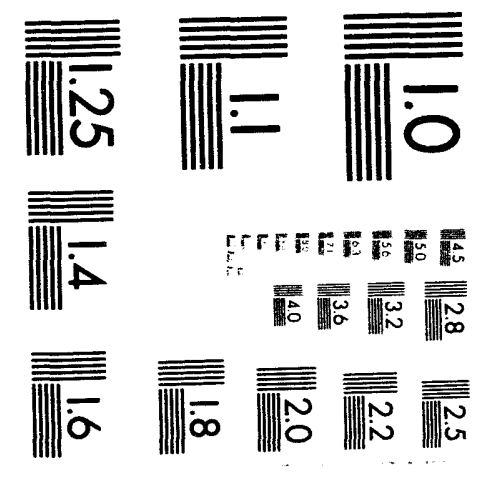



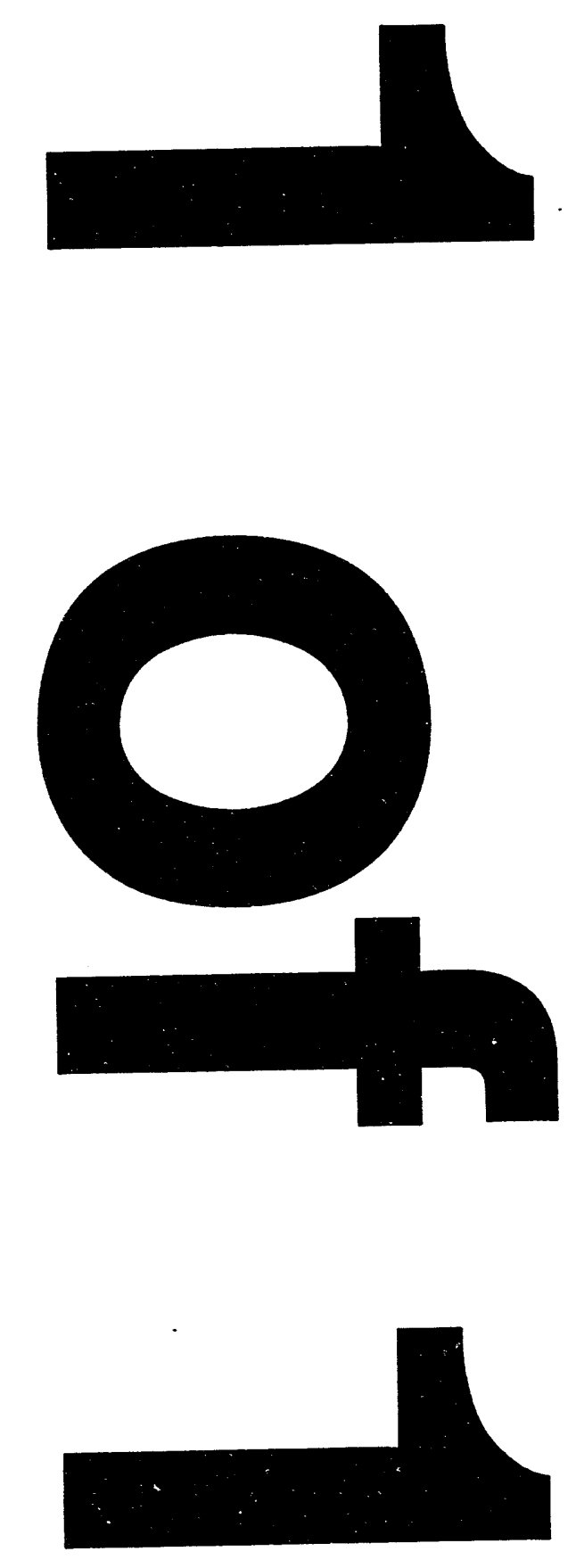
BNL-60019

Informal Report

Limited Distribution

SNS-3

\title{
Beam Scraping Efficiency in the 5MW Spallation Neutron Source
}

\author{
A.J. Stevens
}

\section{RHIC Project}

\section{BROOKHAVEN NATIONAL LABORATORY}

UPTON, LONG ISLAND, NEW YORK 11973 


\section{Introduction}

Numerical calculations have been performed to evaluate the performance of a simple beam scraping system in the lattice of a rapid cycling $(30 \mathrm{~Hz})$ proton accelerator designed to be a high intensity spallation neutron source. ${ }^{1,2}$ The assumption has been made that beam loss will be dominated by of capture inefficiency at injection (600 MeV kinetic) which is simulated as described below. Comparison is made with a "sample calculation" intended to illustrate betatron (beam halo) scraping at full energy which was assumed to be $3 \mathrm{GeV}$. The parameters of the lattice are those of the design as of April, 1993.

The results described here are restricted to a geometry wherein the primary scraper is treated as an aperture in the $X$ (horizontal) coordinate. Evaluation of the efficacy of two dimensional scrapers, ${ }^{3}$ or scraping systems which employ magnetic fields remain as topics for further study.

The purpose of this study was to determine to what extent beam losses can be confined to a local region of the proposed lattice.' The remainder of this note describes the general methodology employed (Section II), the rf capture beam loss simulation (Section III), and the beam halo growth "sample calculation" (Section IV). Section V briefly summarizes the results of this study.

\section{Methodology}

The evaluation consisted of three stages. The first of these is a Monte Carlo program which simulates orbit growth, the general features of which are described in the next paragraph. When the orbit impinges on either the front face or the side of the scraper, the coordinates $\left(x, x^{\prime}, y, y^{\prime}, z, \Delta p / p\right)$ are stored in a file for tracking through the scraper block. This tracking was performed by utilizing the computer program ELSIM. ${ }^{4}$ If the orbit was not absorbed in the block, its coordinates on emerging the block were written to another file. Finally, these outscattered orbits were tracked through the lattice for a small distance in order to determine possible locations for secondary absorbers.

The fundamental assumption in the first stage of the evaluation is that orbit growth occurs only in the $x$ coordinate and can be described by the equation:

$$
x_{s}(t)=a \times \sqrt{\beta_{s}} \times \cos \left(\psi_{s}\right)+\chi_{p} \frac{\Delta p}{p}
$$

where either $\Delta p$ or $a$ is changing with time. Initially, i.e., at $t=0$, the horizontal betatron amplitude $a$ is sampled from a distribution appropriate for a linear machine with emittance $\varepsilon_{\mathrm{N}}=$ $\mathrm{K} /(\beta \gamma)$ where $\mathrm{K}=200 \times(\beta \gamma)_{600 \mathrm{MeV}} \mathrm{mm}$ mrd. The one sigma value of $a$ is $\sigma_{\varepsilon}=\sqrt{\varepsilon_{N} / 6}$. The initial value of $\Delta p / p$ is sampled from a parabolic distribution with an rms value of $0.3 \%$ $\sqrt{p_{\text {Max }} / p}$. For the capture loss simulation, eqn. (1) has an additional $(\Delta p / p)_{\mathrm{eff}}$ term which 
increases with time whereas for the halo growth calculation, $a$ becomes $a(t)$. These orbit growth terms are discussed more fully below. Although the subscript $s$ in eqn. (1) denotes any position in the lattice, in practice $x$ and $x^{\prime}$ were evaluated only at the upstream edge of the assumed scraper position once per revolution, and a test was made of whether the orbit struck either this edge or the face of the scraper block somewhere along its length. If the scraper was struck, a $y, y^{\prime}$ coordinate was generated from the appropriate vertical lattice functions and the orbit written to a file as described above. If the scraper was not struck, the orbit was tracked for another revolution, i.e., either $a$ or $\Delta p / p$ was increased and $\psi \rightarrow \psi+2 \pi v_{x}$. The horizontal tune, $v_{x}$ was taken as $8.65 \pm x$ where $X$, which represents a tune spread due to space charge, is chosen randomly between 0 and 0.1 .5

\section{Momentum Scraping Simulation}

Orbits not captured at injection behave as particles which are increasingly off-momentum relative to the synchronous orbit as the B field rises. Hence the scraper must be at a location of relatively high dispersion. The location chosen is shown schematically below.

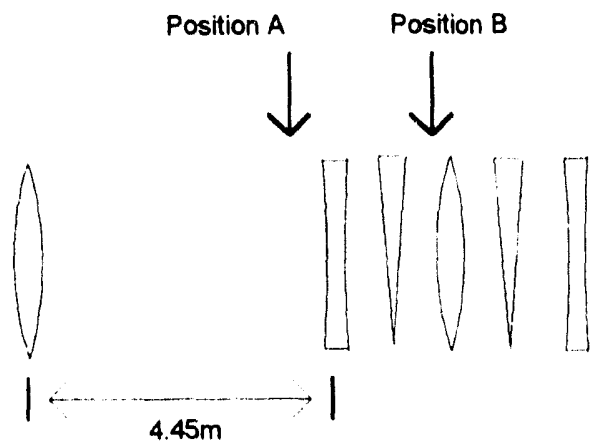

Fig. 1 Lattice elements (lengths not shown) near the momentum scraper

[ The positions indicated are discussed below ]

At $0.5 \mathrm{~m}$ downstream of the exit of the first focusing quadrupole shown, the horizontal lattice functions are the following: $\beta_{x}=13.528 \mathrm{~m}, \alpha_{x}=2.08664, \chi_{p}=2.3631 \mathrm{~m} /(\Delta \mathrm{p} / \mathrm{p})$. The scraper was assumed to be steel and $35 \mathrm{~cm}$. long. The field was assumed to rise from zero to its maximum value in $1 / 60$ seconds as a sine wave. This gives a total $\Delta p$ equal to the intrinsic $\Delta p$ plus:

$$
\left(P_{\operatorname{Max}} / 2\right) \times[1+\sin (60 \pi t-\pi / 2)]
$$


with $t$ in seconds. The scraper edge was somewhat arbitrarily placed at $5 \sigma$ which is $-10.618 \mathrm{~cm}$ for the assumed injection emittance and the minus sign indicates that the scraper is on the lowmomentum (inside) side of the ring.

The scraping efficiency is defined as the fraction of the incident particles which are absorbed. Fig. 2 shows this quantity as a function of the tilt angle $(d x / d z)$ of the scraper generated from 1000 Monte Carlo events at each angle. The angle " 0 " on this figure is not with respect to the beam line but with respect to a "nominal" tilt of

$$
X_{e} \frac{\chi_{p}^{\prime}}{\chi_{p}}+\sigma_{\varepsilon}\left(\frac{\alpha_{x}}{\sqrt{\beta_{x}}}+\sqrt{\beta_{x}} \frac{\chi_{p}^{\prime}}{\chi_{p}}\right)
$$

where $X_{e}$ is the horizontal coordinate of the upstream edge of the scraper and the lattice functions are evaluated at this point. This result follows from solving eqn. (1) for an effective $\Delta p / p$ at the maximum of the betatron oscillation, $(\cos \psi=1)$, then plugging the result in the derivative of (1) to get $x^{\prime}$ for the "typical" betatron amplitude of $\sigma_{\varepsilon}$

Figs. 3 and 4 show $x, y$ scatterplots of the outscattered orbits at the positions indicated in Fig. 1 which are the results of the last part of this simulation. The boundaries of the scatterplot are $\pm 11 \mathrm{~cm}(\mathrm{x})$ by $\pm 7.25 \mathrm{~cm}(\mathrm{y})$ which is the vacuum chamber aperture. At position B (see Fig. 1) only 17 of the original 1000 orbits remain within the physical aperture. Clearly secondary absorbers should be placed downstream of the primary scraper. The actual design of such a system, as well as integrating such a system with rf cavities which also "compete" for space in the straight sections is beyond the scope of this note. The primary conclusion here is that $\geq 98 \%$ of loss due to capture inefficiency can be confined to a local region of the lattice.

The capture loss is not the only source of loss at injection. For example, the (very well defined) stripping inefficiency in the $H^{-}$injection should also be considered. Again, this consideration is deferred to a time when the accelerator reaches a more detailed stage.

\section{Betatron Amplitude Growth}

It is not really possible to simulate loss due to betatron amplitude growth because a valid simulation would require a detailed model of the growth mechanism and no such model is available. [Or likely to ever be available; if a mechanism which causes beam growth is known, "before the fact", the accelerator design would likely be changed to defeat such a mechanism.] At high energy proton colliders such growth (beam halo growth) is slow; one measurement at the SPS $^{6}$ gives an average halo growth of $5 \sigma / \mathrm{sec}$. at a lateral position of $6 \sigma .^{7}$ Such a growth rate would not be observed in the $162 / 3 \mathrm{msec}$. rise time of this accelerator. Consider, for example, an orbit on the "edge" of the beam $(2.5 \sigma)$ at injection. For this orbit to grow to $5 \sigma$ at $3 \mathrm{GeV}$ requires a growth rate of $150 \sigma / \mathrm{sec}$. where the latter $\sigma$ is the $3 \mathrm{GeV}$ beam size. It is $\boldsymbol{a}^{\prime}$ priori likely, therefore, that the rapid cycling nature of this machine will limit non-injection energy losses to very small values. 


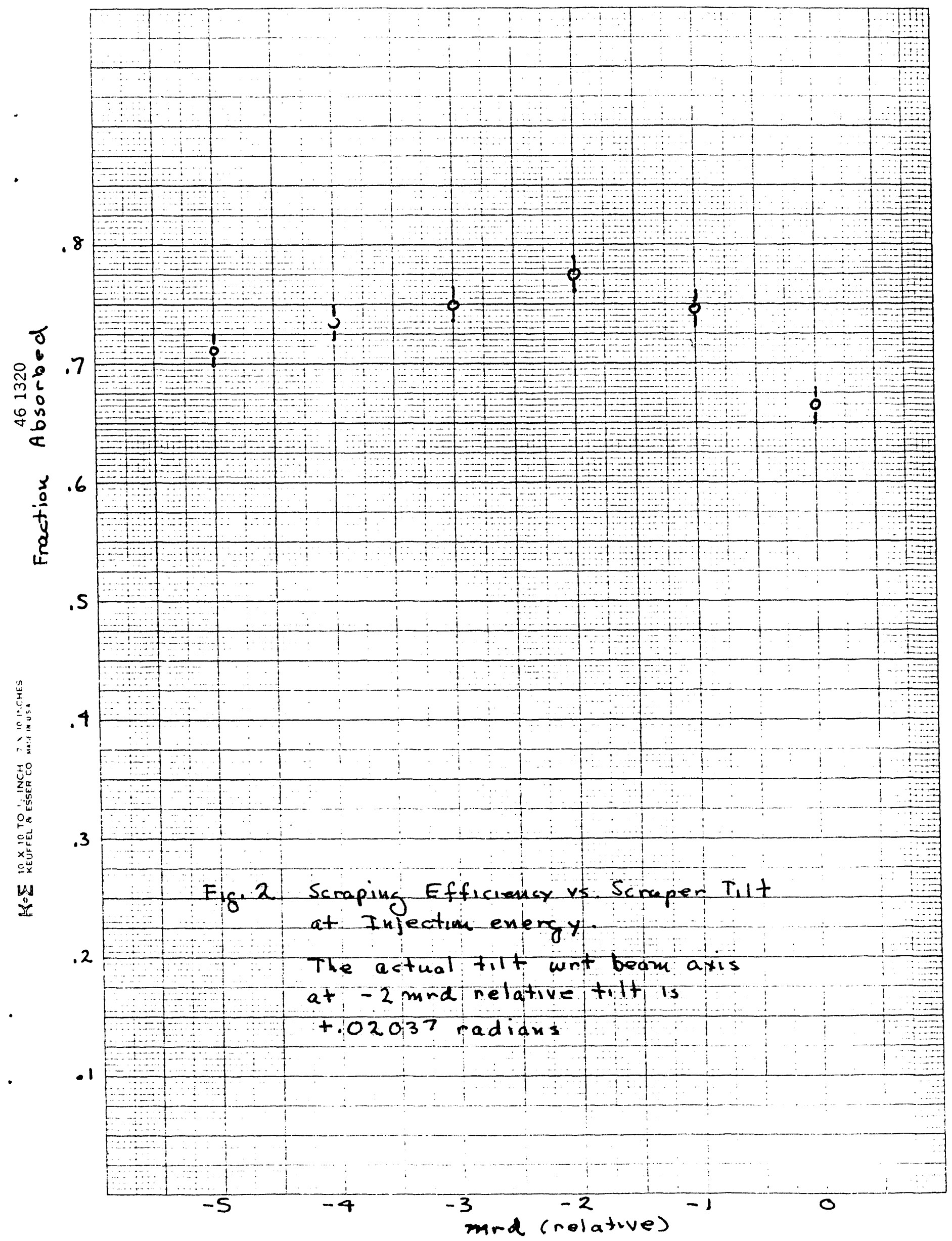


$x$ isy at End 5

HBODK $\cdot I D=-4$

DATE E9107793

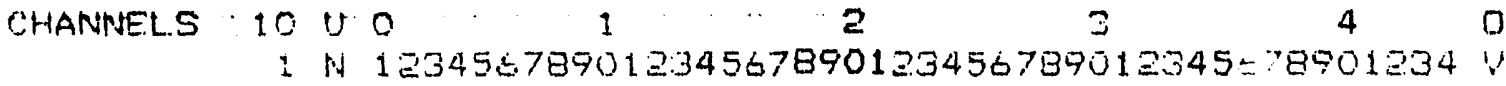

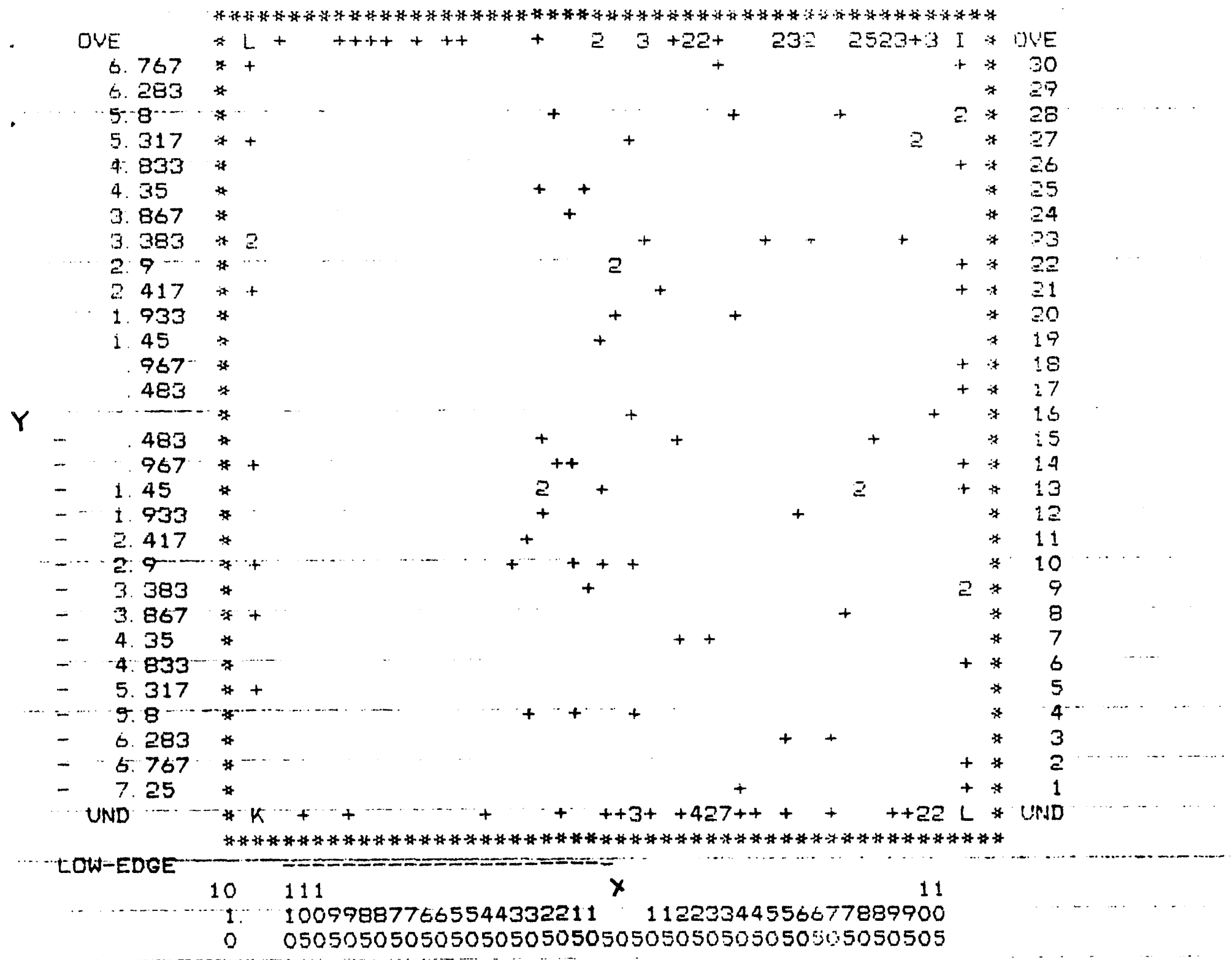

ENTRTES

PLOT

* SATURATION AT=

*-SCALE ; ; $2 ; 3 ;$

* STEP $=1.00$

31

A, B,

STATISTICS

* MINIMUM $=0.000 E+00$

\begin{tabular}{ccccc}
21 & $I$ & 43 & $I$ & 18 \\
\hline 7 & $I$ & 45 & $I$ & 15 \\
\hline 20 & $I$ & 34 & $I$ & 21
\end{tabular}

Fig. 3 . X (sm.) vs. $Y(\mathrm{~cm})$ at Position A (see Fig. 1) of Outscattered Orbits $\left(600\right.$ Mev-Sptimal $\left.T_{1} 1 t\right)$

49/1000 within physical aperture 
$\therefore$ XYY at Enrio

HBOOK ID $=\quad \theta$

ENTE 29:07:53

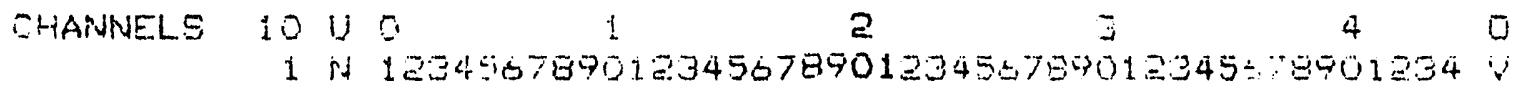

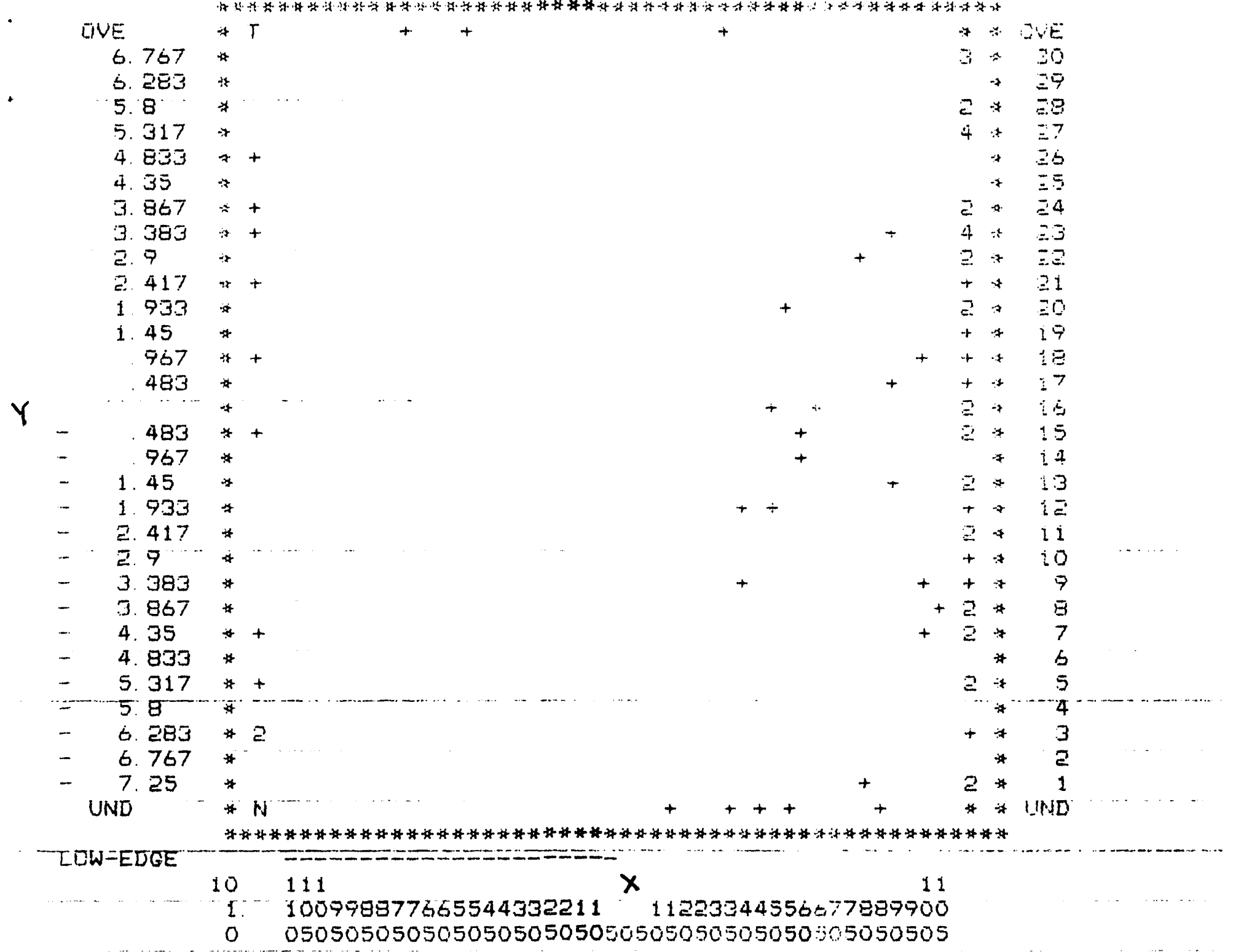

Fig. $4 . X(\mathrm{~cm})$ vs. $Y(\mathrm{~cm})$ at Position B $(F / g .1)$ of Outscattered Orbits (600 Mev-Optimal Tilt) $17 / 1000$ within physical aperture 
A "sample calculation", rather than a simulation, was nonetheless performed in order to crudely estimate the potential behavior of a simple scraper at $3 \mathrm{GeV}$. We assume that a programmed orbit bump could keep the tail of the beam a constant $5 \sigma$ distance from a primary scraper edge during the acceleration cycle. For this calculation the first phase of the Monte Carlo was performed at a kinetic energy of $3 \mathrm{GeV}$, and an $a(t)$ value (see eqn. (1)) of $150 \sigma(3 \mathrm{GeV}) / \mathrm{sec}$. was assigned. In this case, the $5 \sigma$ position of the scraper was at $5.99 \mathrm{~cm}^{8}$

The scraping efficiency vs. scraper tilt for this case is shown in Fig 5. The "o's" in this Figure pertain to the lattice section sketched in Fig. 1, while the "+'s" relate to a location with the structure $Q F \cdot L \cdot Q D \cdot L \cdot Q F \cdot L \cdot Q D$ where $L$ represents the long $4.45 \mathrm{~m}$ drift length. In this latter location, the lattice functions 0.5 meters downstream of the first quadrupole are the following: $\beta_{x}$ $=13.69 \mathrm{~m}, \alpha_{\mathrm{x}}=1.99, \chi_{\mathrm{p}}=.112$. The lattice functions are very similar in the two locations except that the dispersion is smaller. As is evident, the difference in scraping efficiency in the two locations modeled is very small.

Little difference is also seen when outscattered orbits are tracked. Figs 6 and 7 show the $x, y$ scatterplots at the same locations as Figs. 3 and 4 in the injection energy case. Although secondary absorbers could intercept some of the orbits near the edges of the aperture, it is nonetheless true that $15-20 \%$ of the total orbits scraped escape the local region. There are two reasons for this. The assumed transverse growth of $150 \sigma / \mathrm{sec}$ produces less displacement on the front edge of the scraper than the magnetic field rise at the injection energy and therefore less probability of interaction. Secondly, the higher energy particles which escape have undergone less multiple scattering in the absorber.

\section{Summary}

A scraping simulation of injection orbits which are not captured by the if shows that a simple primary scraper with secondary absorbers located near the primary scraper should intercept approximately $98 \%$ of such beam loss.

A programmed bump could keep the beam "tail" a fixed distance from a scraper during the accelerator ramp in order to scrape growing beam halo. Such halo is expected to be small because of the fast cycling time. At the top energy of $3 \mathrm{GeV}$, which is expected to represent the worst case as regards scraping efficiency, approximately $85 \%$ of the loss is expected to be confined to the local region where scraping occurs.

A number of issues remain for further study. These include the placement of apertures to intercept additional losses at injection, integration of the scraping system with other components (especially rf cavities) of the lattice, local radiation damage effects from beam loss, and evaluation of the potential of more sophisticated scraping systems. 
.8

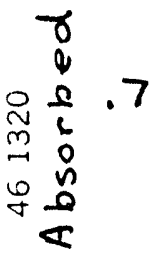
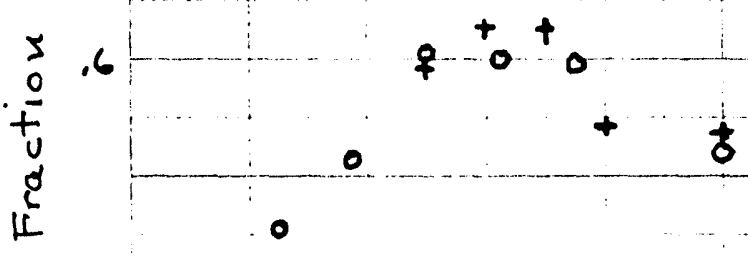

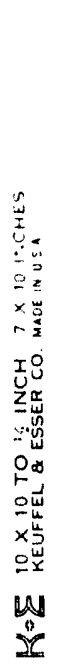

.5

○

$\circ$

.4

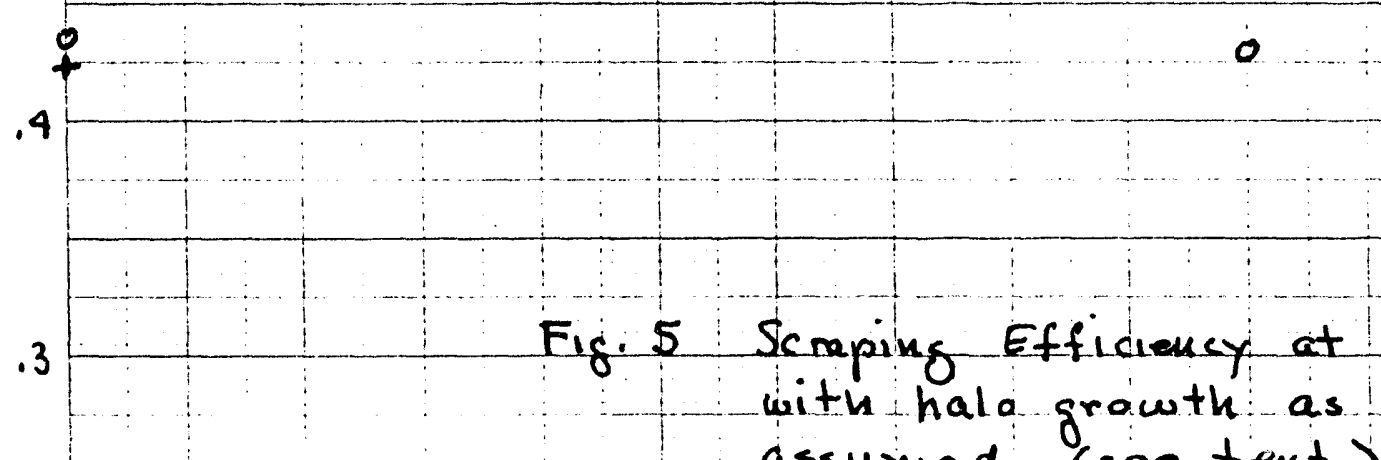

assumed (see text.)

"O's" are for Fig .l lattice location

"t's" are for lattice location

.2 with low dispersion

Errors (not show ix) are as

in Fig. 2

$\bullet 1$

0

1.0

2.0

mind (relative) 
$x$ xy at End

HEOOK $\quad \mathrm{IO}=4$

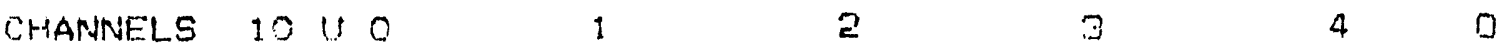

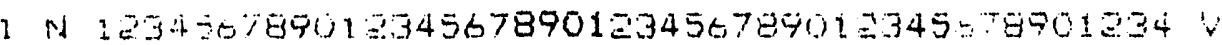

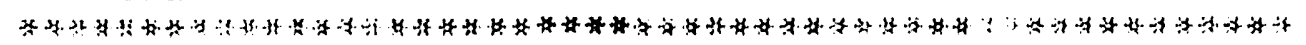

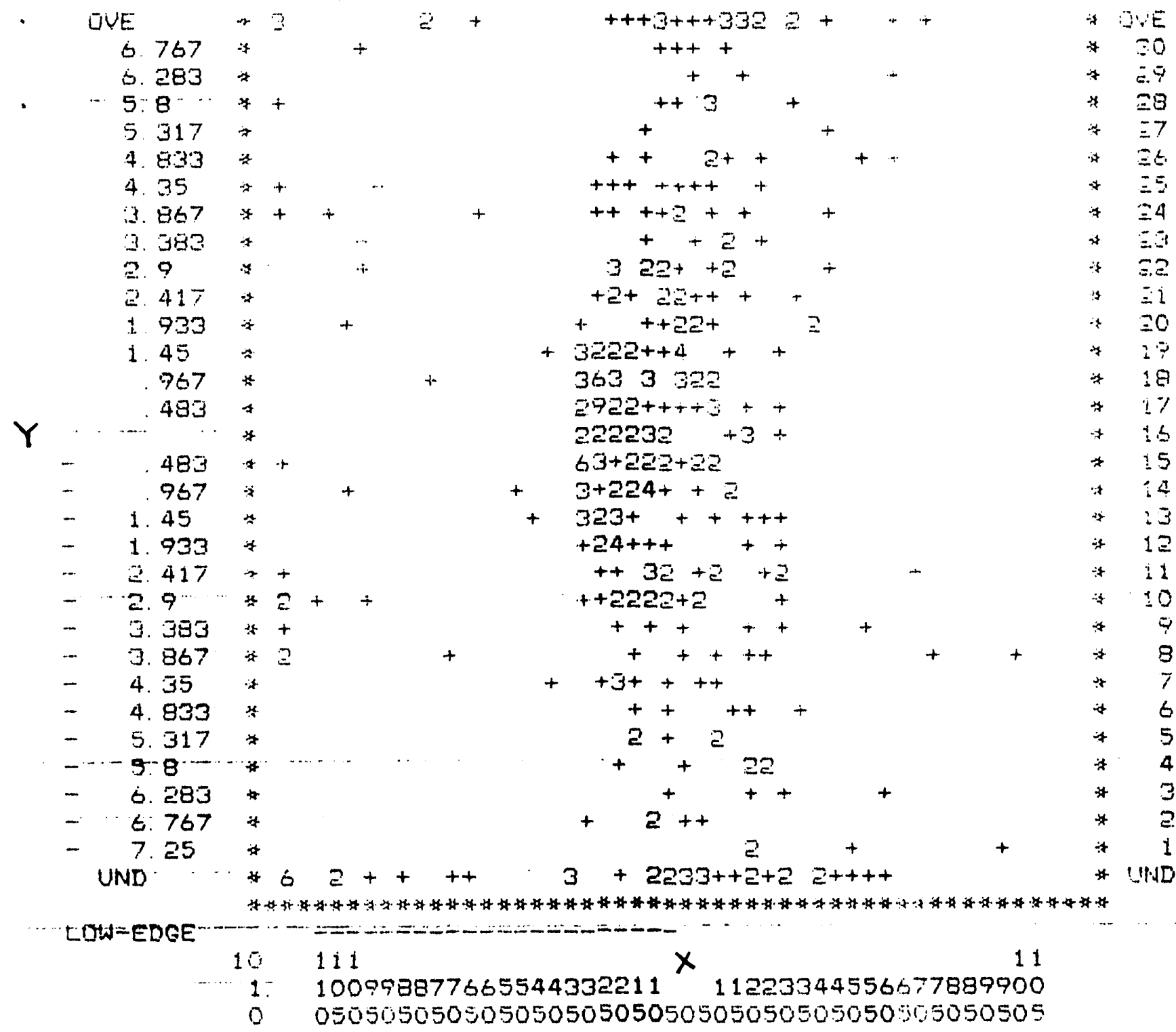

$*$

* ENTRTES

394

PLDT

* saturatign at=

* SCALE $.+, 2,3$,

$+, 2,3,:, A, B$,

31

* STEP $=1.00$

- MINIMIJHa $=0.000 E+00$

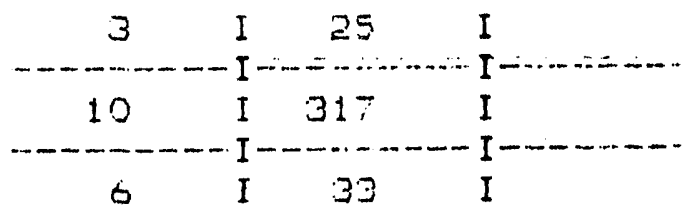

Fig. 6 X(cm.) vs. Y (cm.) at Position A (Fig. I) of outscattered orbits (3GeV-Optimal T,1t) $317 / 1000$ within physical aperture 
HBOOK

$I D=$

8

DHTE O4.08/93

CHANAMELS $10 \cup 0$

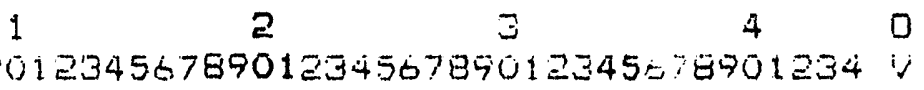

UVE

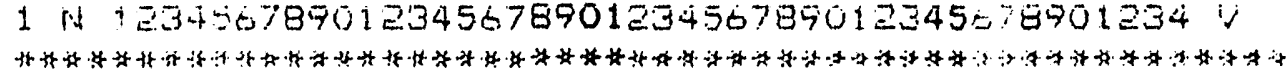

6. 767

6. 283

5. 8

5. 317

4. $833 *+$

4. $35 *+$

3. $867 * \equiv$

3. $383 * 2$

2. 9

E. 417

1. 933

1. $+8$

$+2++0+3 V E$
$++2+30$

967

$483 * 2$

Y

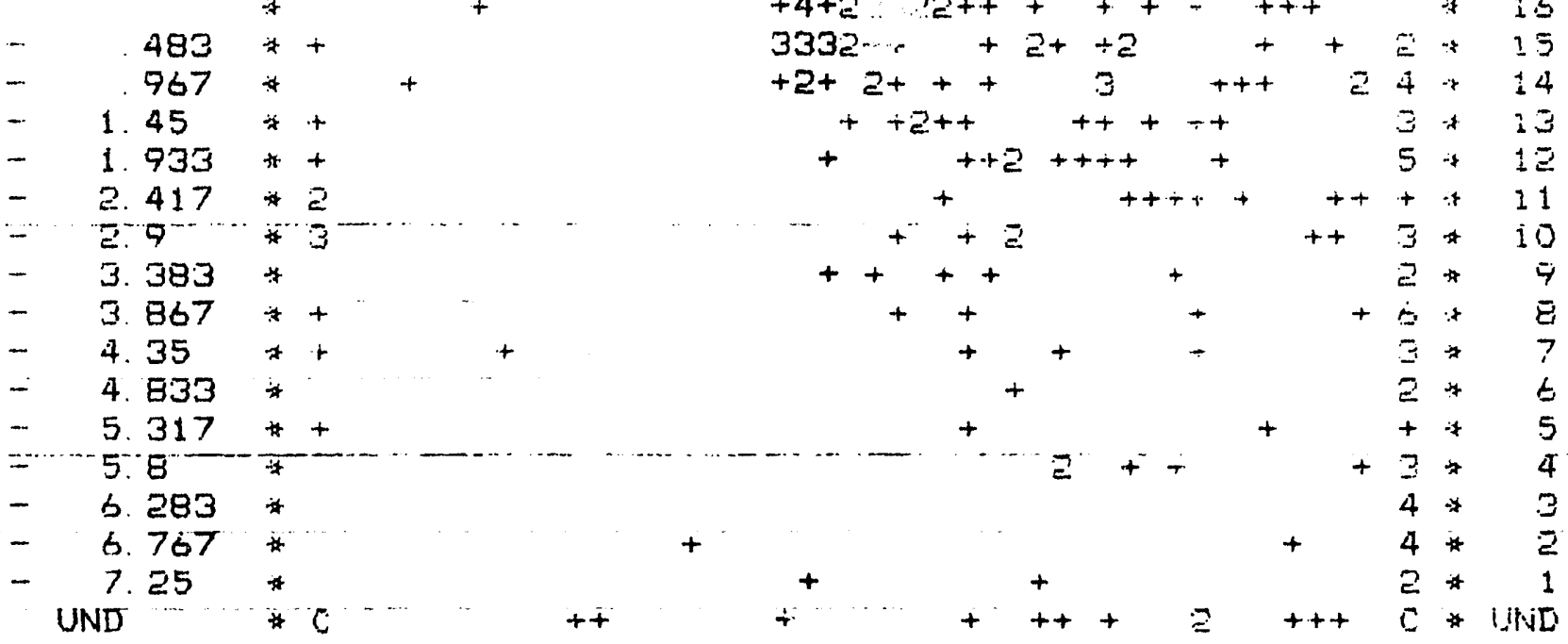

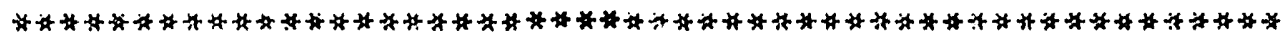

LDW-EDGE

$$
\begin{array}{llrr}
10 & 111 & 11 \\
1 . & 100958877665544332211 & 11223344556677887900 \\
0 & 05050505050505050505050505050505050505050505
\end{array}
$$

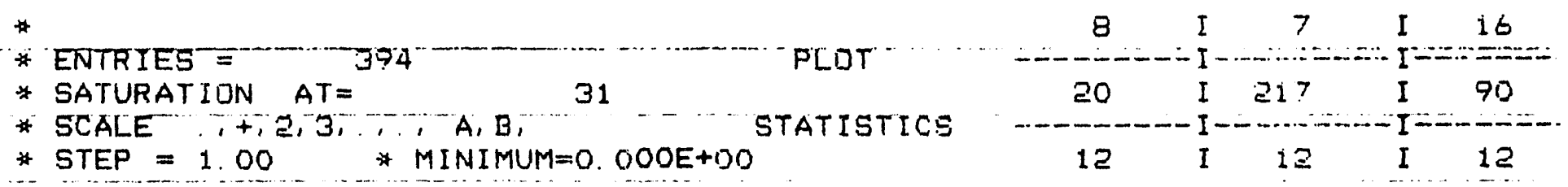

Fig. 7 X(cm) ys. $Y(\mathrm{~cm})$ at Position B ( $\left.F_{1 g .1}\right)$ of outscattered orhits ( 3 GeV-Optimal Tilt) $211 / 1000$ within physical apenture. 


\section{Footnotes/References}

1. A. Ruggiero and A. van Steenhergen, "Baseline Design of a 5 MW Spallation Neutron Source," BNL-49246, Inf. Rep. SNS-1 (1993)

2. L.N. Blumberg, "Preliminary Report on the BNL Spallation Neutron Source Design Study." BNL-49274 Inf. Rep. SNS-2 (1993).

3. P.J. Bryant, "Some Advantages of Inclined Collimators," CERN SL/92-24 (AP) (1992).

4. A. Van Ginneken, "Elastic Scattering in Thick Targets and Edge Scattering," Phys. Rev. D37, 3292 (1988).

5. S. Ruggerio, private communication.

6. L. Burnod and J.B. Jeanneret, "Transverse Drift Speed measurement of the Halo in a Hadron Collider," Proceedings of the Workshop on Advanced Beam Instrumentation, A. Ogata and J. Kishiro, Eds., April 22-24, 1991.

7. The speed of halo drift is position dependent. As the orbits grow, larger non-ideal magnetic field components are encountered which causes accelerated growth.

8. The assumed orbit bump was, in fact, ignored. In principle, the scraper should have been physically at the assumed injection energy value, and the beam "brought to the scraper." Including the bump in the simulation would have added a complexity that is not justified by the illustrative nature of this "sample calculation." 

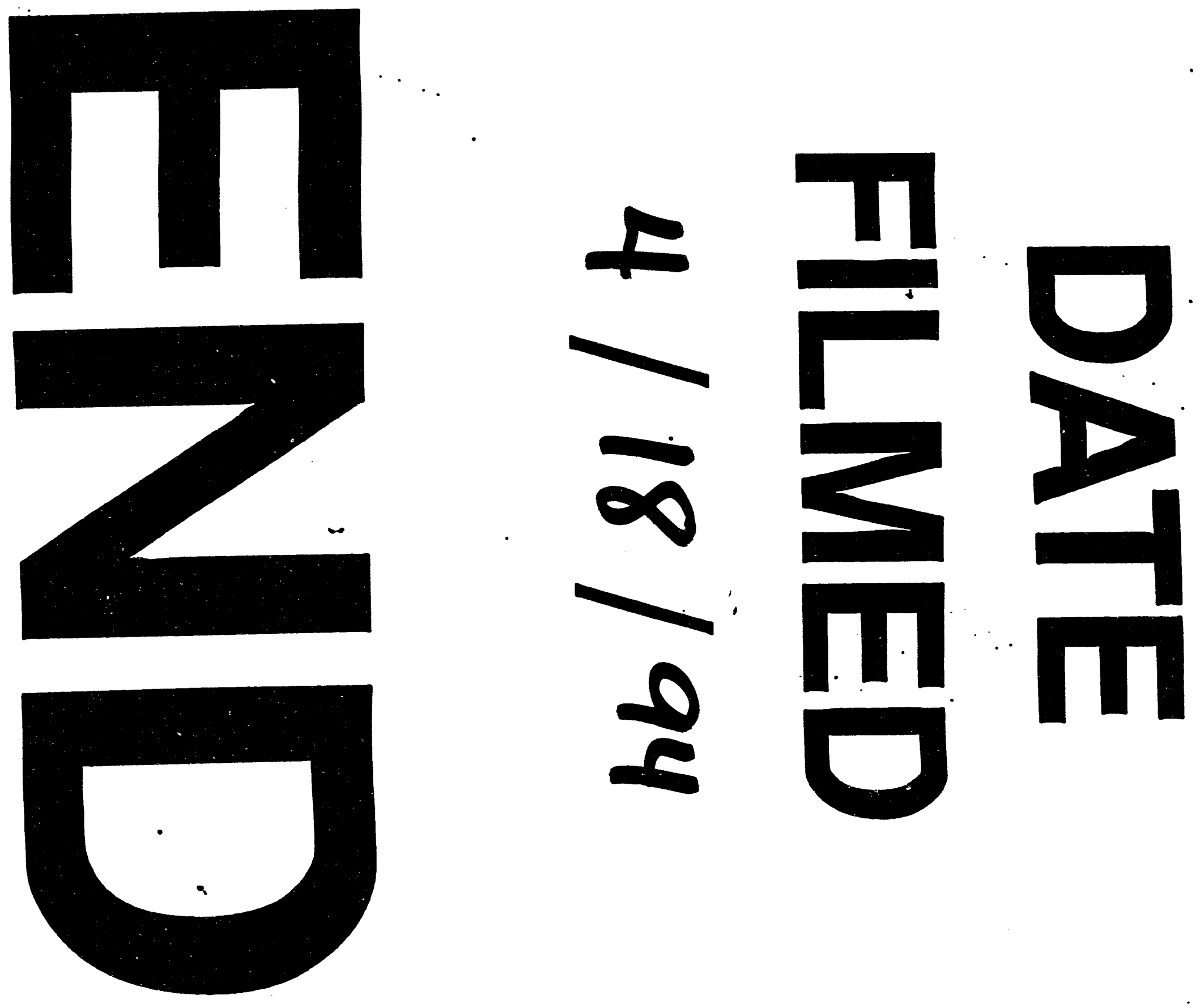


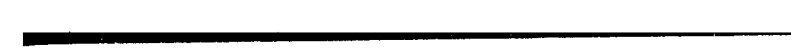$$
1
$$

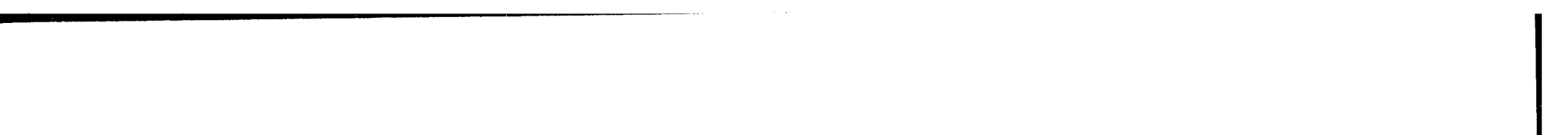

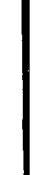

